durch Punction dor Pleuraböble and Aspiration der in ihr enthaltenen Luft, wie dies von Stephen Mackenzie vorgeschlagen und auch ausgeführt wurde ${ }^{1}$ ), nur dann geschehen dürfen, wenn durch den hochgradigen intrapleuralen Druck lebensgefährliche Erscheinungen bedingt würden (vergl. auch Walter a. a. 0. S. 241), aber auch dann müsste dies mit grösster Vorsicht geschehen und nnter Vermeidung der Möglichkeit von Hustenanfällen.

\title{
2. Ueber einen Fall von ulceröser Entzündung der Trachea und des linken Bronchus in Folge eines Aneurysmas des Aortenbogens mit Durchbruch dieses in die Trachea.
}

Am 24. Oetober 1888 secirte ich folgenden interessanten Fall.

D., Charles-Théodore, 48 Jahre alt, Tüncher, wurde am 21. October in die medicinische Klinik aufgenommen und starb daselbst bereits am 23 .

Derselbe war bis vor einem Jabr vor seiner Aufnahme in's Spital nie krank gewesen. Von diesem Zeitpunkt ab hatte er, angeblich in Folge einer starken Erkältung heftige Hustenanfälle und immer stärker werdende asthmatische Beschwerden. Er hatte dabei stets das Gefühl, als ob ihm Schleimmassen im Halse sässen, die er nicht auszuwerfen vermochte. Der schlaf war immer gut, aber schnarchend und Morgens beim Erwachen hatte er It ühe, etwas Schleim herauszubringen. Bei der geringsten Anstrengung und besonders beim Treppensteigen kam er ganz ausser Athem. In letzter Zeit bustete er ein wenig. Am 16. October musste or heftigen Herzklopfens wegen die Arbeit aussetzen und sogar nach Hanse gebracht werden. Am 21. spuckte er Blut. Erscheinungen von Bleivergiftung waren niemals vorhanden gewesen.

Beim Eintritt in's Spital bot der Kranke ausser seinen Athembeschwerden nichts Besonderes dar. Die Herzdämpfung war etwas verbreitert und der Spitzenstoss nach links von der Mamillarlinie im 6. Intercostalraum fühlbar. Abnorme Herzgeräusche oder Arytbmie waren nicht vorbanden. Es bestand inspiratorischer und exspiratorischer Stridor und zwar besonders stark über der Trachealbifurcation. Ueber der Lunge fand sich rauhes Athmen, hier und da etwas Pfeifen, sonst aber nichts Besonderes. Die Percussion ergab ebenfalls nicbts Besonderes. Die Stimme war normal. Es bestand chronischer Rachenliatarh, das Schlucken war nicht erschwert, Appetit, Verdauung und Stublgang waren gut. Harn normal.

1) The Lancet. 1871 . Vol. II. p. 259. 
Am 23. October Nachts $12 \frac{1}{2}$ Uhr bekam der Kranke plötzlich einen heftigen Erstickungsanfall, er spuckte ungefähr $50 \mathrm{ccm}$ bellrotbes Blut aus und starb nach ungefähr 10 Jinuten.

Die klinische Diagnose war: Aortenaneurysma, obwohl trotz sorgfältigen Untersuchens darauf keine Erscheinungen eines solchen wabrgenommen werden konnten.

Anatomische Diagnose: Aneurysma der aufsteigenden Aorta und des Aortenbogens mit Perforation in die Trachea. Ulcerationen der Trachealund linksseitigen Bronchialwand in Folge von Compression. Hydropericard. Herzhypertrophie, alter myocarditischer Heerd in der linken Herzwand zunächst der Herzspitze. Lungenemphysem, Lungenhyperämie und -ödem an der Basis. Starke Hyperämie des Gehirns und überbaupt aller Organe, Plethora.

Sectionsbefund: Grosser, woblgebauter, sehr musculöser Mann. Haare und Augen schwarz, Gesichtsfarbe blass, an den abhängigen Theilen zahlreiche, grosse Todtenflecke, starke Todtenstarre. Ungebung des Mundes, der Nase und linke Wange mit eingetrocknetem Blut bedeckt.

Dolichocephaler Schädel. Hirnbäute und Gehirn durchaus normal, nur ausserordentlich byperämiscb. Die prall mit Blut erfüllten Gefässe baben vollkommen normale Wandungen.

Unterbautfettgewebe gut entwickelt, die sehr kräftige Musculatur dunkelroth gefärbt. Rippenknorpel verkalkt. Linke Lunge gut, rechte weniger gut zurückgezogen. Das Pericard liegt in grosser Ausdebnung vor. Das etwas nach links verlagerte Herz ist sehr gross, seine Spitze wird durch den linken Ventrikel gebildet. In den Pleurasäcken ist keine Flüssigkeit vorbanden, in Pericardialsack finden sich zwei Gläser gelbliche, klare Flüssigkeit. Pericard nermal, Epicard verdickt, besonders hinten und an der Basis. Sämmtliche sehr vergrösserte Herzhöhlen enthalten viel flüssiges Blut und grosse, rothe Gerinnsel.

Linke Lunge nicht verwachsen, gross, aufgebläht, schwer, am Rande etwas emphysematisch. Aus dem Bronchus entleert sich viel biutige Flüssigkeit. Durch die unveränderte Pleura hindurch gewahrt man viele, verschieden grosse, rothe Stellen, anscheinend bedingt durch in den Alveolen vorhandenes Blut; dieselben sind an der Lungenbasis reichlicher vorbanden und grösser als in den oberen Partien. Auf dem Schnitt zeigt der Unterlappen grösseren Blutreichthum als der Oberlappen, ferner findet sich daselbst ziemlich starkes Oedem. Die rechte Lunge ist binten verwachsen, sonst verhält sie sich ganz wie die linke.

Die Mund- und Halsorgane werden zusammen mit dem Herzen herausgenommen.

Herz durchweg sehr vergrössert. Rechter Vorbof und Herzohr stark erweitert, ihre Wandung durchweg verdickt. Die Vorhofswand ist zunächst dem Herzohr $5 \mathrm{~mm}$ dick, das Endocard ist durchweg verdickt, das Foramen ovale ist geschlossen. Rechter Ventrikel sehr gross. Derselbe hat zunächst der A. pulmon. einen inneren Uufang von $110 \mathrm{~mm}$ und wisst von der A. 
pulmon. $a b$ bis zur Eerzspitze $110 \mathrm{~mm}$, von letzterer bis zur Tricuspidalinsertion $90 \mathrm{~mm}$. Die A. pulmon. hat etwa $10 \mathrm{~mm}$ über den Klappen einen Umfang von $80 \mathrm{~mm}$. Zunächst der Arterie bat die vordere Herzwand eine Dicke von 6, etwa in der Mitte eine solche von $10 \mathrm{~mm}$. Die PulmonalkJappen und Tricuspidalsegel sind normal. Linker Vorhof ist ebenfalls sehr weit und seine Wandung verdickt, dieselbe misst $4 \mathrm{~mm}$. Linker Ventrikel stark erweitert; er hat einen inneren grössten Umfang von $160 \mathrm{~mm}$ und misst von den Aortenklappen bis zur Spitze 95, von der Mitralinsertion bis zur Spitze $110 \mathrm{~mm}$, die grösste Wanddicke beträgt $22 \mathrm{~mm}$. Aortenklappen und Mitralsegel normal. Die Aorta hat gerade über den Klappen einen Umfang von $90 \mathrm{~mm}$. Von da ab bis zur $A$, subclav. sin. reichend findet sich ein den ganzen Aortenumfang einnehmendes Aneurysma. Sein grösster Umfang beträgt $260 \mathrm{~mm}$, seine Wand ist durchweg endarteritisch verdickt und nach links und hinten mit röthlich gefärbten, ziemlich dicken Thromben bedeckt. Jenseits des aneurysmatischen Sackes hat die Aorta einen Urfang von $75 \mathrm{~mm}$, die Intima ist von da ab nur an wenigen Stellen etwas verdickt.

Die Zunge ist mit blutigem Scbleim bedeckt, der Oesophagus normal.

Kehlkopf und Luftröhre enthalten viel blutigen Schleim und Blutgerinnsel. Der Kehlkopf ist normal gebaut, seine Schleimhaut unverändert, die Knorpel sind verknöchert. Die Luftröbre hat durch eine sebr hochgradige Verringerung ihres Querdurchmessers eine säbelscheidenartige Form erhalten. Diese Querverengerung ist oben weniger stark als unten. Hier etwa $20 \mathrm{~mm}$ nach aufwärts von der Bifurcation ab ist ibre Lichtung in einer Ausdehnung von $12 \mathrm{~mm}$ auf ein Minimum reducirt. Sie hat an dieser Stelle ausserdem nicht nur eine Biegung nach rechts erfahren, sondern ist auch zugleich noch etwas um ihre Axe gedreht worden, so dass ihre rechte Wand nach hinten, die linke aber nach vorn gewendet ist. Bier ist ihre Lichtung auch vollständig mit dunklen Blutgerinnseln erfüllt. Nach Entfernung des Schleimes und der Blutgerinnsel zeigt sich, dass der 6., 7. und 8. Trachealring vorn in der Mittellinie und zwar in einer etwas schief ron oben rechts nach unten links zu verlaufenden Linie gebrochen sind, ohne dass die sie bedeckende Schleimbaut irgend eine besondere Veränderung darböte. Die Trachealschleimbaut ist durchweg stark geröthet. Besonders stark aber ist sie es vom 9. Trachealring $a b$ bis zur Bifurcation und von da bis in den Anfangstheil der Bronchien. Ausserdem ist sie in der gleichen Ausdebnung ziemlich stark verdickt, am meisten nach hinten zu. Vom 9. Trachealring ab nach unten $\mathrm{zu}$ beginnt auch die hochgradige Verengerung der Luftröbre und soweit diese reicht, finden sich in der den knorpligen Theil der Trachealwand bedeckenden verdickten Schleimbaut ziemlich grosse und tiefe Geschwüre. Rechts findet sich ein solches von $20 \mathrm{~mm}$ Durchmesser. Dasselbe bat verdickte Ränder und einen unebenen, wie zerrissenen Grund, die Knorpelringe sind aber nicht ganz von Weicbtbeilen entblösst. Nach unten gegen den rechten Bronchus $z u$ ist die Schleimhaut ganz besonders stark verdickt und gerade vor dem Bronchus findet sich eine querverlaufende Leiste und 
nach beiden Seiten davon je eine kleine buchtige Vertiefung, die aber wit vollkommen glatter Schleimbaut ausgekleidet sind. Auf der entgegengesetzten linksseitigen Trachealwand, dem erwähnten Geschwür gerade gegenüber, nur etwas höber hinaufgehend, findet sich eine sehr starke Verdickung der Schleimbaut und in ihr drei Geschwüre, ein oberes, hinteres und unteres. Die beiden ersteren berübren sich nach hinten zu. Das untere ist von dem oberen durch ein $S$, von dem binteren durch eine $4 \mathrm{~mm}$ breite, sehr verdickte Schleimbauturäcke geschieden. Das obere Geschwür misst in der Quere 10, in der Höhe $7 \mathrm{~mm}$, es ist etwas trichterförmig vertieft und sein Grund ist mit rothem Granulationsgewebe bedeckt. Das hintere hat eine quadratische Form und einen Durchmesser von $11 \mathrm{~mm}$. Das untere, fast bis zur vorderen Mittellinie reichende Geschwür ist rundlich und bat einen Durchmesser von $7 \mathrm{~mm}$. Nach unten $\mathrm{zu}$ ist die Schleimbaut $5 \mathrm{~mm}$ weit abgehoben. Das Geschwür erstreckt sich vom unteren Rand des 10. bis zum unteren Rand des 13. Knorpelringes. Bei rorsichtigem Sondiren des Geschwärgrundes nimmt man wahr, dass der untere Rand des 13 . Knorpels vom Perichondrium entblösst ist. Die Weichtheile zwischen ihm und dem nächstfolgenden Knorpelring fehlen an einer, $36 \mathrm{~mm}$ oberhalb der Bifurcation gelegenen, Stelle vollständig and man gelangt von da in einen Kanal, in welchen man die Hohlsonde bequem einführen kann. Dieselbe erscheint bei vorsichtigem Vorschieben im Aneurysmensack, in deren hinterer Wand $40 \mathrm{~mm}$ von der A. subclav. sin. und ebenso weit von der A. anonyma entfernt sich eine etwas unregelmässig rundliche Oeffnung von $7-8 \mathrm{~mm}$ Durchmesser findet, aus der sie hervorkonmt Bei genauerem Zuseben zeigt es sich aber, dass diese Oeffnung durch ein ihrem unteren Rand in der ganzen Quere anhaftendes ebenso grosses Stück Arterienwand ausgefültt wird. Nach links $\mathrm{zu}$ ist die Arterienwand 5, nach links und oben $\mathrm{zu} 11 \mathrm{~mm}$ weit von der dahinter liegenden Luftröhrenwand abgelöst und gerade hier findet sich auch der vom Perichondrium entblösste Knorpelring. Nach rechts zu hat eine Ablösung der Arterienwand nicht stattgefunden. Weiter nach unten ron der letzterwähnten perforirenden Trachealulceration und auf derselben Seite, nur mehr nach hinten $z u$, gerade äber dem 15 . Knorpelring, findet sich noch ein $4 \mathrm{~mm}$ im Durchmesser haltendes seichtes Schleimbatgeschwür. Gerade über der Bifurcationsstelle und an dieser selbst ist die Schleimbaut ebenfalls ziemlich stark verdickt und erodirt.

Im linken Bronchus, gerade unterbalb seinem Ursprung, findet sich an der hinteren Wand ein ovales Geschwür mit einem Querdurchmesser von 13 und einem Höhendurchmesser von $9 \mathrm{~mm}$. Dasselbe ist ungefähr $3 \mathrm{~mm}$ tief, sein Rand fällt ringsum steil $a b$, ist unten und seitlich nicht verdickt und ist nicht abgelöst, während er oben verdickt und etwas unterminirt ist. Der Geschwürsgrund ist vollkommen eben und glatt, die Knorpel sind nicht entblösst.

Milz gross, ausserordentlich blutreich, normal.

Beide Nebennieren rollkommen normal.

Beide Nieren gross, Kapsel leicht abziehbar. Oberfäche glatt, überaus hyperämisch, desgleichen die sonst vollkommen normale Schnittfäche. 
Magen und Darmaual volkowmen nomal.

Geber sehr gross, sehr blutreich, aber obne jegliche wabraenbare Veländerung.

Sämotliche grosse Gefässe, Arterien sowohl wie Venen, eathalten ansserordentlich viel Blut.

Der 7. Trachealring, also der mittlere von den drei fracturiten, wurde von hinten her abgelöst, in Celloidin eingebettet und geschnitten. Derselbe ist ziemlich stark verkalkt, besonders zunächst seiner äusseren, weniger stark zunächst seiner inneren Oberfläche. In der Mitte findet sich nur unbedeutende Kalkablagerung um die Kapseln. Ganz nach aussen und innen, ungefähr so weit die olastischen Fasern in ibn eindringen und die Knorpelzellen kleiner und scheinbar jünger sind, findet sich gar keine Kalkeinlagerung. Es zeigt sich auf den Schnitten, dass der Bruch fast durch den ganzen Knorpel hindurchgeht. Innen erreicht er das Perichondrium und ist dieses stellenweise in ihn eingellemmt. Aussen ist dies nicht der Fall, weil er nicht ganz bis zum Perichondrium reicht. Die Bruchränder sind nicht glatt, sondern etwas zackig, die Zacken aber wie durch Resorption leicht abgerundet, die Knorpelsubstanz zunächst dem Bruchrand durchscheinender, etwas zellenärmer und leichter färbbar als das übrige Knorpelgewebe. In einem Schnit findet sich zwischen dem etwas abgelösten inneren Perichondrium und dem Knorpel ein freies, rundlicbes, sebr durchscheinendes Knorpelstückwhen mit nur zwei, je eine Zelle enthaltenden Kapseln. Zeichen eines Blutargusses seitens des Perichondriums finden sich nirgends vor.

Vom Grund der verscbiedenen Geschwüre der Luftröhre wurde gleich triseh etwas weggenommen und untersucht. Es fanden sich hier überall in Zerfall begriffene Zellen und Zellendetritus, Fettröpfchen und elastische Easern.

Nach der Erbärtung wurden Schnitte der verdickten Trachealschleimbaut znnächst dem rechten Bronchus angefertigt. Hier zeigt sich nun, dass die Yerdickung durch hochgradige kleiazellige Infiltration, die einen fast adenoiden Charakter hat, bedingt ist. Stellenweise erstreckt sie sich bis in's Perichondrium, ist aber zunächst der Schleimhautoberfläche ganz besonders stark. Das Grundgewebe ist bier nicht oder nur mit Wühe wahrzunehmen, whinend es in der Tiefe ganz gut erkennbar ist, besonders einzelne Fettzellen treten hier dentlich hervor'. Die Oberfäche ist vielerorts noch mehr oder weniger gut mit Epithel bedeckt. Da wo dasselbe fehlt, hat sie eive zerrissene Beschaffenheit. In nächster Umgebung solcher Stellen finden sich anch in besagtem kleinzelligen Gewebe hier und da Mikrokokkencolonien, die Riesenzellen täuschend ähalich sehen. Anderweitig kommen solche nicht vor. Das Knorpelgewebe zeigt hier ein fast durchweg normales Verhalten. Stärkere Verkalkung fehlt, nur um verbältnissmässig wenige Knorpelkapseln indet sich eine geringfügige Kalkablagerung. Ferner finden sich bier und da in der inneren Hälfte des Knorpels braunes, königes Pigment enthaltende Knorpelzellen. Mitunter sind auch die Gefässe des inneren Perichondriums stärber mit Blut gefüllt. Frische Blutergüsse oder Reste von älteren sinu 
aber hier nicht anffudhar. Das äussere Perichondrium hat ein volkommen normales Aussehen.

Vorliegender Fall ist hauptsächlich dadurch interessant, weil er in unzweifelhafter Weise erkennen lässt, wie die Aortepperforation, welche doch als die eigentliche Todesursache angesehen werden muss, zu Stande kam.

Die an der Trachea vorgefundenen Veränderungen, theilweise Drehung um ihre Axe, säbelscheidenartige Verengerung und ulceröse Entzündung, müssen wohl als Folgezustände der aneurysmatischen Erweiterung des Aortenbogens angesehen werden. Die Verengerung bestand sicherlich schon vor der Entzündung, ohne jedoch vorerst wesentliche Beschwerden zu verursachen. Diese stellten sich erst mit der Schleimhautentzündung ein und diese mag sehr wohl nit der vom Kranken erwähnten starken Erkältung ibren Anfang genommen haben. Einmal vorhauden, konnte sie nicht mehr gut rückgängig werden, sondern musste wegen der immer zunehmenden Verbiegung und Verengerung der Trachea durch Druck von aussen und wegen der im Inneren sich anhäufenden und festhaftenden Schleimmassen stetig zunehmen. Da wo die einander immer näher kommenden Schleimhautoberflächen sich, wenn auch nur zeitweilig und vorübergehend, berührten, musste an der hochgradig entzündlich infiltrirten und erweichten Schleimhant erst Erosion und dann tiefergehende Ulceration entstehen. Eines der so entstandenen Geschwüre, das untere links, beschränkte sich sogar nicht auf die Schleimhaut, sondern ging tiefer bis auf den Knorpel, legte durch Zerstörung der Knorpelhaut diesen frei, und zwischen dem 13. und 14. Knorpelring weitergehend gelangte dasselbe rasch auf die hier der vorderen Trachealwand innig anhaftende hintere Aortenwand. Diese wegen ihres festen Gefüges und ihres geringen Gefässreichthumes setrte dem weiteren Vordringen des entzündlich-eitrigen Prozesses einen vorerst unüberwindbaren Widerstand entgegen. Eine nothwendige Folge hiervon war, dass derselbe in der Breite that, was er in der Tiefe nicht thun konnte, und dass sich somit in der Adventitia der Aorta ein kleiner Senkungsabscess ausbildete, der nothgedrungen die Widerstandskraft der Aortenwand an der betreffenden Stelle verringern musste. Eine weitere Folge hiervon 
war dann, moghicherweise gelegentlich ane stakeren in nstron. gung oder eines heftigen Hustenanfalles, Aass die ohnedies schon brüchigere Aortenwand daselbst einriss und der erste nicht sehr bedeutende Blutaustritt statt hatte. Dass dieser erste Bluterguss war in der Form eines einfachen Blutspuckes auftrat, mag vielleicht seinen Grund darin haben, dass zuerst nur ein Einriss stattfand, durch welchen nur wenig Blut austreten konnte. Erst als dann zwei Tage später an der Bruchstelle der aussen blossgelegte Wandbezirk an noch zwei Seiten einriss und die Oeffsung grösser wurde, konnte auch eine grössere Menge Blut austreten und den Tod herbeiführen. Aber auch dieses Mal war die Menge des nach aussen ergossenen Blutes nicht sehr gross, and zwar wahrscbeinlich weil das an einer Seite noch festhaftende Bruchstück der Aortenwand gewissermaassen ein Ventil bildete, weil der von da in die Trachea führende Kanal sehr eng war und weil das in die hochgradigst verengte Trachea geZangte Blut sofort gerann und dem nachströmenden Blut einen Damm entgegensetzte. Wenn trotz des verhältuissmässig geringen Blutaustrittes der Tod so rasch eintrat, so ist dies bei den gegebenen Veränderungen an der Trachea leicht erklärlich. In Folge der Verstopfung der Trachea und zum Theil auch der Bronchien durch das in sie gelangte Blut musste in der ohnehin schon hyperämischen Lunge ein acutes Oedem sich ausbilden and in Folge dessen der Tod eintreten.

Gegen die etwaige Annahme, dass erst ein Aortenriss stattgefunden und das ausgetretene Blut sich allmählich, innerhalb zweier und einem halben Tag, einen Weg nach dem Tracheallumen gebahnt habe, spricht: das Fehlen eines Aneurysma dissecans zwischen der hinteren Aortenwand und den sie umgebenden Organen, der vom Perichondrium entblösste Trachealknorpel, die geringe Weite des zwischen Aorta und Trachea vorhandenen Kanales und seine Einmündung in eines der Schleimhautgeschwüre.

Das Vorhandensein des vom Perichondrium entblössten Knorpels ist für sich allein schon ein zwingender Beweis für die Richtigkeit der ersten und gegen eine solche der zweiten Annahme. Ein mechanischer Reiz, wie das aus der Aorta ausgetretene und, durch die Weichtheile sich Bahn brechende Blut 
jhn ausüben konnte, vermochte keine Ablösung der Knorpelhaut zu bewirken, dies konnte nur ein länger dauernder eitriger Entzüudungsprozess, der hier auch in der That vorhanden war.

Als besondere Eigenthümlichkeit dieses Falles wäre noch die vorgefundene Fractur des 6.-8. Knorpelringes zu erwähnen. Ob dieselbe auf Rechnung des durch das Aortenaneurysma ausgeübten Druckes gesetzt werden darf, wage ich um so weniger zu entscheiden, als weiter unten, wo dieser Druck sicherlich noch stüker war, keine Fracturirung statt hatte. Nach F. Riegel sollen in seltenen Fällen durch den Druck ,vergrösserter Schilddrüsen" „auf die verknöcherten Knorpel spontane Fracturen entstehen" ${ }^{\text {I }}$. In vorliegendem Falle war jedoch die Verkalkung nur mässig stark und gerade da, wo sie am stärksten war, aussen, war der Knorpel nicht fracturirt. Der mikroskopische Befund scheint jedoch immerhin dafür zu sprechen, dass der Bruch der Knorpelringe schon zu Lebzeiten stattfand, denn nur so kann sich die Abrundung der Zacken der Bruchflächen und die grössere Transparenz und Zellenarmuth des ihr zunächst gelegenen Knorpelgewebes erklären.

Ich habe vorliegenden Fall so ausführlich mitgetheilt, weil die Zahl der veröffentlichten Fä]le von Aortenaneurysmen mit Perforation in die Trachea nicht gar gross ist und besonders weil die dabei vorkommenden anatomischen Veränderungen in der Regel nicht eingehender behandelt sind. Im Allgemeinen wird zwar angenommen, dass bei der Perforation von Aneurysmen in die Luftwege eine bis auf die Arterienwand sich fortsetzende Verschwärung oder Nekrose der Tracheal- oder Bronchialschleimhaut der Perforation vorausgeht. Prüft man aber die in der Literatur niedergelegten einschlägigen Beweise genauer, so wird man bald wahrnehmen, dass dieselben nicht ausreichend sind eine von $R$. Thoma vertretene andere Ansicht $z u$ widerlegen. Derselbe, gestützt auf seine sorgfältigen und dankenswerthen "Untersuchungen über Aneurysmen" ${ }^{2}$ ), glaubt sich in vielen Fällen davon überzeugt zu haben, dass beim Durchbruch aneurysmatischer Säcke in die Bronchien oder in den

I) In H. v. Ziemssen, Handb. d. spec. Pathol. u. Therap. 2. Auf. Bd. 3. I. 2. Hälfte. S. 231.

2) Dieses Archiv. 1888. Bd. 112. S. 266. 
Oesophagus erst eine Verlöthung der beiderseitigen Wandungen and dann eine gemeinsame Dehnung und Verdünong der Scheidewand erfolgt, woraufhin diese einreisst. Hiervon ganz verschieden sind die Ansichten der älteren Forscher. So sagt ъ. B. schon Hodgson, in den von mir beobachteten Fällen, in denen Aneurysmen auf der Oberfläche des Körpers oder in denen mit Schleimmembranen ausgekleideten Höhlen geborsten waren, war die Oeffnung immer die Folge von chronischer Verschwärung ${ }^{61}$ ). Im Gegensatz hiervon soll nach ihm (ebd.) der Durchbruch von Aneurysmen in seröse Säcke durch Dehnung, Verdünnung and Einreissen erfolgen. J. F. H. Albers erwähnt unter den Ursachen, die eine Perforation der Trachea bedingen können, ${ }_{3}$ das Aneurysma arcus aortae, welches, wie überall, so auch hier, durch Druck und seine Pulsation die härteren Theile der Trachea atrophirt und zugleich eine verschwärende Entzündung in der Schleimbaut verursacht, da das Aneurysma nur den unteren Theil der Trachea erreicht, fiuden sich die Geschwüre gleich oberhalb der Bifurcation" ${ }^{2}$ ). Letzteres war auch hier der Fall und ebenso in den Fällen von Pridie ${ }^{3}$ ), Bögehold ${ }^{4}$ ) und Heath ${ }^{5}$ ). Auch C. Rokitansky bemerkt bei Besprechung der ulcerösen Prozesse grösserer Arterien, dass dieselben "vielen lebensgefährlichen Blutungen zu Grunde liegen: es gehören hierher die Perforationen der Aorta von der Trachea, vom Oesophagus her " ${ }^{6}$ ), und A. Förster sagt "Perforation einer Arterie von aussen nach innen wird durch ulceröse Destruction bewirkt, die sich von den umgebenden Geweben und Organen auf die Ar. terienwände fortsetzt, es gehören hierher die bekannten Perforationen der Aorta bei Geschwüren der Trachea"7 $)$.

Die Richtigkeit der Ansicht von Hodgson und der anderen arwähnten Autoren wird durch vorliegenden Fall vollauf bestätigt

1) Krankh. d. Art. u. Ven., deutseh von F. A. Koberwein. Hannover 1817. S. 128.

3) Erläuter. z. d. Atlas d. path. Anatom. 1839. 1. Bd. II. Abth. S. 116.

3) Edinb. med. Journ. 1862. VII. p. 1161 .

4) Berl. klin. Wochenschr. 1881, S.58.

5) Transact. of the pathol. Soc. Vol. IX. p. 95. - Ref. in Sehmidt's Jabrb. 1889. Bd.110. S. 246.

Lehrb. d, path. Auatom. 3. Aufl. 1856. 2. S. 303.

Handb. d. path. Anatom. 2. Aufl. 1863. Bd.2. S. 741. 
und er ist um so beweisender für dieselbe, als die vorgefundenen durch das Aneurysma veranlassten Schleimhautgeschwüre sich nicht nur auf der dem Aneurysma zugekehrten, sondern auch auf der entgegengesetzten Wand der Trachea und des linken Bronchus vorfanden. Ohne bestreiten zu wollen, dass die von Thoma angenommene Entstehungsweise des Durchbruchs aneurysmatischer Säcke vorkommen kann, bin ich doch fest überzeugt, und eigene Beobachtungen von solchen Perforationen in die Bronchien bestätigen es mir auch, dass die bei diesem Falle festgestellte die. weitans häufigere ist. Dies ist eigentlich auch ganz natürlich, denn in Folge der Compression der Luftwege entstehen innerhalb ibrer Wandung an den betreffenden Stellen Circulationsstörungen, die den Boden für die Entzündung vorbereiten. Diese stellt sich dann aber um so rascher ein und führt um so leichter zu Geschwürsbildung, als sich ebenda Schleimmassen und Fremdkörper anhäufen und als fortdanernder Entzündungsreiz wirken. Sobald aber die entzündliche Einschmelzung über die Knorpelringe hinausgeht, muss durch die bis in die Adventitia sich fortsetzende eitrige Entzündung erst eine Schwächung und dann eine Zerreissung der Aneurysmawand und somit ein Blutaustritt erfolgen. 